\title{
2564. The research for partitioned algorithm of coupling of elastic pipe and cross-flow in a cylinder
}

\author{
Qian Bei Yue', Ri Zhi Dong' ${ }^{2}$ Ju Bao Liu ${ }^{3}$ \\ College Mechanical Science Engineering, Northeast Petroleum University, \\ Hei long-jiang, Daqing, 163318, China \\ ${ }^{1}$ Corresponding author \\ E-mail: ${ }^{1}$ zlgbb529@126.com, ${ }^{2} 940762474 @ q q . c o m,{ }^{3} j s l x 2000 @ 163 . c o m$
}

Received 14 September 2016; received in revised form 26 December 2016; accepted 19 January 2017 DOI https://doi.org/10.21595/jve.2017.17731

Check for updates

\begin{abstract}
In the marine flatbed, nuclear power stations and oil fields, the marine risers and heat exchangers are induced vibrations and collision, even caused failure with cross flow. This is a typical problem of bundles vibration in fluid-structure interaction. In this paper, the elastic pipe was separated into several beam elements. And the dynamical equation was adopted to describe the structure. The fluid domain of cylinder was separated into solid elements. And the computational fluid dynamics equation was adopted to describe the fluid. At the fluid-structure coupling interface, the formulas of displacement, velocity and load was derived and the corresponding convergence criterion was also derived. Thus, the partitioned coupling algorithm was established for the elastic pipe and the cross-flow in cylinder. The example showed that, comparing to the results of monolithic coupling algorithm, the error was small. Thus, it proved that the correctness of the partitioned coupling algorithm in this article. And it will provide an effective computational method for vibrations and collision of bundles in a more complex fluid domain.
\end{abstract}

Keywords: partitioned coupling algorithm, monolithic coupling algorithm, elastic pipe, fluid-structure interaction.

\section{Introduction}

In the marine flatbed, nuclear power stations and oil fields, the marine risers (seen as Fig. 1) and heat exchangers $[1,2]$ are induced vibrations and collision, even caused failure with internal flow and external flow. This is a typical problem of bundles vibration in structure-interaction coupling. It related to multiple movable interfaces and multiple physical fields and which was difficult to calculate. Most of the results were studied the tube vibrations with internal flow and the vibration law was analyzed in longitudinally. M. Ragulskis [3] adopted harmonic balance method to study the non-Newtonian fluid flow in longitudinally vibrating tube. The tube vibrations induced by internal flow and the tube was a non-deformable rigid body. So far, the numerical methods for the tube vibrations with cross flow (external flow) are monolithic coupling algorithm and partitioned coupling algorithm [4-7]. In monolithic coupling algorithm, equations are solved simultaneously for fluid and structural domain. The advantage is the high accuracy $[8,9]$. The disadvantage is that because the equations are integrated for fluid and structure and the solution efficiency is very low. Especially, it is not suitable for problems of large-scale and more complex domain. In partitioned coupling algorithm, equations are solved respectively. Within each time step or iteration, the information is transferred at the coupling interface. And the coupled solution is achieved. The advantage is that the solving range is wide and easy to conduct. The disadvantage is that the difference of grid density for fluid domain and structural domain at the coupling interface and which causes the grid do not match $[10,11]$. Therefore, it need data transfer at the coupling interface. And it is the first to solve the problem in partitioned coupling algorithm. Comparing to the results of monolithic coupling algorithm, the error was small. The research outcome will make commutations of vibration and collision for bundles possible in a more complex fluid domain. 

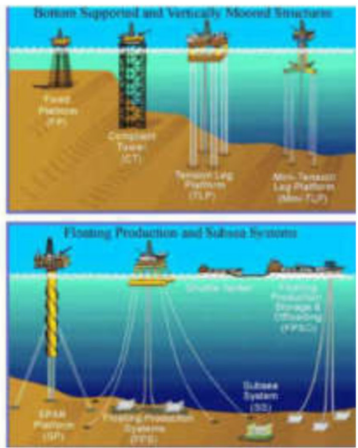

a) The marine risers in the ocean

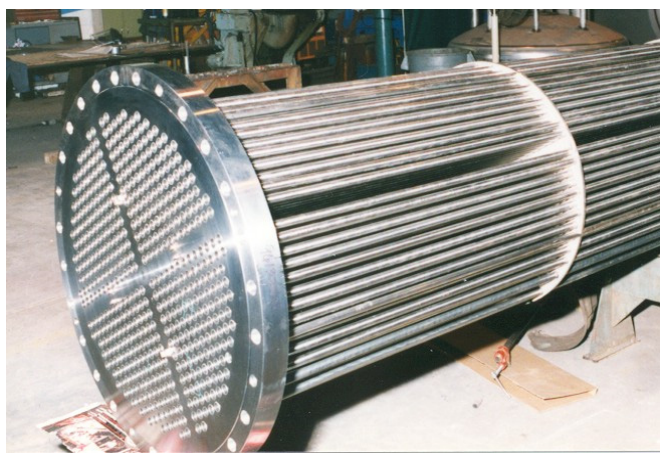

b) The heat exchanger tubes

Fig. 1. The tubes in engineering

\section{The mechanics model of the coupling of elastic pipe and cross-flow in cylinder}

The fluid and elastic pipe was selected as research object in cylinder. The coupling mechanics model of the elastic pipe was set up. As shown in Fig. 2, the model was divided into two domains: the structural domain $\Omega_{s}$ and the fluid domain $\Omega_{f}$. The assumptions were used as followed.

(1) The fluid medium is water. And it assumes the water is sticky and incompressible.

(2) The solid is assumed to be perfectly elastic body. And the elastic tube is prismatic. The cross section is always circular.

As shown in Fig. 1, the boundary of cylindrical entrance was velocity. And it was denoted by $\Gamma_{D}^{f(1)}$. The boundary of outlet was pressure and it was denoted by $\Gamma_{N}^{f}$. The boundary that the fluid was flowing along the inner cylinder's wall surface was free slip and it was denoted by $\Gamma_{D}^{f(2)}$. The interface between the fluid and solid interaction was fluid-structure coupling boundary and it denoted by $\Gamma_{F S I}$.

The boundary conditions for the cylinder fluid domain $\Omega_{f}$ were:

$$
\left\{\begin{array}{l}
\dot{\mathbf{d}}_{r}^{f}=\mathbf{n}_{r}^{f} \cdot u, \quad \Gamma_{D}^{f(1)}, \\
\dot{\mathbf{d}}_{r}^{f}=0, \quad \Gamma_{D}^{f(2)}, \\
\mathbf{p}_{r}=\mathbf{n}_{r}^{f} \cdot p_{0}, \quad \Gamma_{N}^{f},
\end{array}\right.
$$

where: $\mathbf{n}_{r}^{f}, \dot{\mathbf{d}}_{r}^{f}, \mathbf{p}_{r}$ were the vectors of normal unit, velocity, pressure, respectively. $u$ was inlet velocity and $p_{0}$ was outlet pressure. The superscripts of $f, s$ were represented fluid, solid respectively.

The structural domain $\Omega_{s}$ was the elastic and the boundary was hinged on both ends.

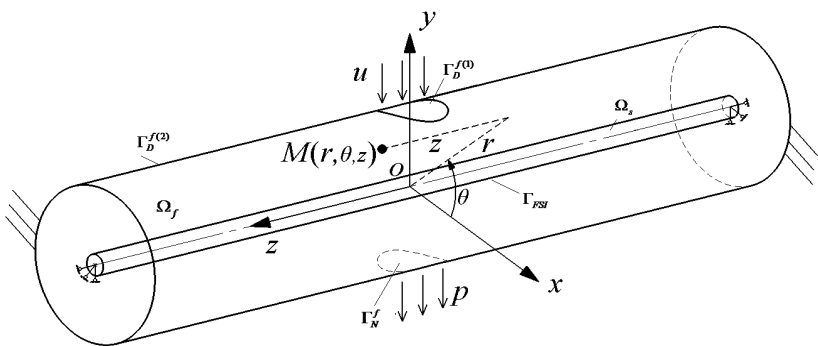

Fig. 2. The mechanics model of the elastic pipe and the cross-flow in cylinder

Supposed that the fluid and solid surface without sliding, the displacement and speed should meet the condition of coordination and the load should meet the condition of load balancing. 
1) The condition of displacement coordination:

$\mathbf{d}^{s} \cdot \mathbf{n}^{s}+\mathbf{d}^{s} \cdot \mathbf{n}^{f}=0$,

where: $\mathbf{d}^{s}, \mathbf{d}^{f}$ were the displacement vectors of solid and fluid respectively. $\mathbf{n}^{s}, \mathbf{n}^{f}$ were the unit displacement vectors of solid and fluid domain respectively. $\mathbf{n}^{s}=\mathbf{n}_{r}^{s}+\mathbf{n}_{\theta}^{s}+\mathbf{n}_{z}^{s}$, $\mathbf{n}^{f}=\mathbf{n}_{r}^{f}+\mathbf{n}_{\theta}^{f}+\mathbf{n}_{z}^{f} \cdot \mathbf{n}_{r}^{s}, \mathbf{n}_{\theta}^{s}, \mathbf{n}_{z}^{s}$ were unit vectors along the $r, \theta, z$ direction.

2) The condition of velocity coordination:

$\dot{\mathbf{d}}^{s} \cdot \mathbf{n}^{s}+\dot{\mathbf{d}}^{f} \cdot \mathbf{n}^{f}=0$,

where: $\dot{\mathbf{d}}^{f}, \mathbf{d}^{f}$ were the velocity vectors of solid and fluid respectively. $\mathbf{n}^{s}, \mathbf{n}^{f}$ were the same as the Eq. (2).

3) The condition of load balancing:

$\boldsymbol{\sigma}^{s} \cdot \mathbf{n}^{s}+\boldsymbol{\sigma}^{f} \cdot \mathbf{n}^{f}=0$

where: $\boldsymbol{\sigma}^{s}, \boldsymbol{\sigma}^{f}$ were the stress vectors of solid and fluid respectively.

\section{The monolithic algorithm of the coupling of elastic pipe and the cross-flow in cylinder}

\subsection{The numerical model of the coupling of elastic tube and cross-flow with monolithic algorithm}

In monolithic algorithm, the fluid and were both divided by solid elements. The numerical model was shown in Fig. 3. The solid elements were adopted eight-node hexahedral element or six-node pentahedral element, as shown in Fig. 4.

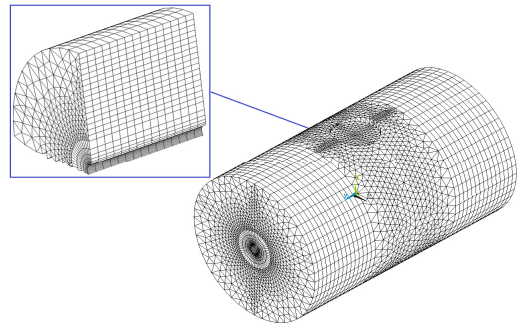

a) The finite element of cylindrical fluid

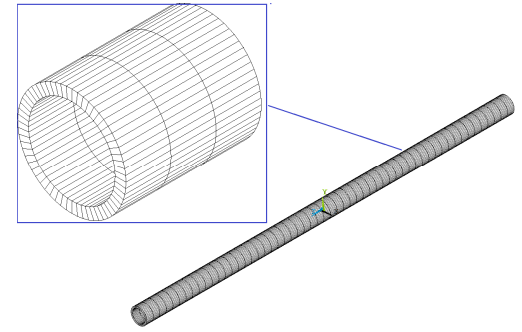

b) The finite element model of elastic tube

Fig. 3. The numerical model of transverse fluid and elastic tube with the method of universe

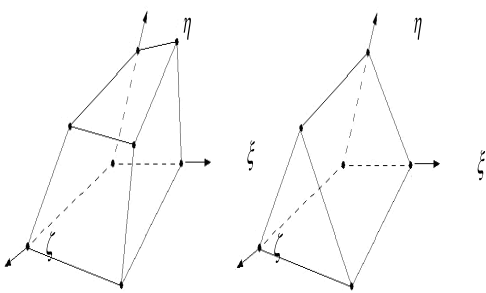

a) Hexahedral element

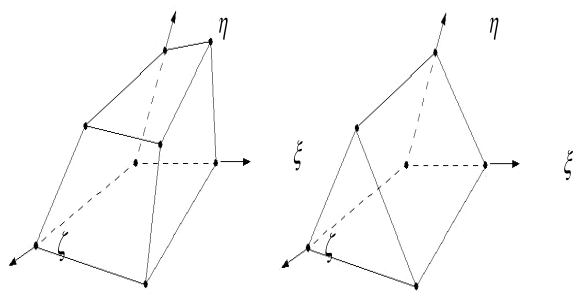

b) Pentahedral element

Fig. 4. Solid element

\subsection{The monolithic algorithm of the coupling of elastic tube and cross-flow}

The fonctionelle of generalized variational principles was as followed in fluid-solid coupled 
system:

$\Pi=\Pi_{0}+Q_{c}+Q_{L}$

where, $\Pi_{0}$ was the fonctionelle of generalized variational principles in fluid and structural domain. $Q_{c}$ and $Q_{L}$ were constraints and external work which cited by the mouthed of Lagrange multiplier in fluid-solid coupled system.

As mentioned above, the fluid and structural domain were disserted by eight-node hexahedral element or six-node pentahedral element. Then the fonctionelle of generalized variational principle was obtained as followed for discrete model of fluid-solid coupling:

$\Pi=\sum_{i=1}^{N^{f}} \Pi_{f}^{(e)}+\sum_{i=1}^{N^{s}} \Pi_{s}^{(e)}+\sum_{i=1}^{M^{c}} Q_{c}^{(e)}+\sum_{i=1}^{M^{c}} Q_{L}^{(e)}$,

where, $N^{f}$ and $N^{s}$ were the numbers of discrete element in fluid and structural domain respectively. $M^{c}$ was the number of nodes on the fluid-structure coupling interface.

Then the finite element equations and constraints were written with matrix form. Then, the dynamic coupling finite element equation was:

$$
\begin{aligned}
& {\left[\begin{array}{cccc}
\mathbf{A}_{\alpha \beta} & 0 & 0 & 0 \\
0 & \mathbf{M} & 0 & 0 \\
0 & 0 & 0 & 0 \\
0 & 0 & 0 & 0
\end{array}\right]\left\{\begin{array}{c}
\ddot{\mathbf{d}}^{f} \\
\ddot{\mathbf{d}}^{s} \\
0 \\
0
\end{array}\right\}+\left[\begin{array}{cccc}
\mathbf{B}_{\alpha \beta \gamma}^{j} \dot{\mathbf{u}}_{\alpha j}+\mathbf{D}_{\alpha \beta}+\mathbf{C}_{11}^{c} & \mathbf{C}_{12}^{c} & \mathbf{C}_{13}^{c} & \mathbf{C}_{\alpha \beta} \\
\mathbf{C}_{21}^{c} & \mathbf{C}+\mathbf{C}_{22}^{c} & \mathbf{C}_{23}^{c} & 0 \\
\mathbf{C}_{31}^{c} & \mathbf{C}_{32}^{c} & \mathbf{C}_{33}^{c} & 0 \\
G_{\alpha \beta} & 0 & 0 & 0
\end{array}\right]\left\{\begin{array}{c}
\dot{\mathbf{d}}^{f} \\
\dot{\mathbf{d}}^{s} \\
\boldsymbol{\lambda} \\
\mathbf{p}
\end{array}\right\}} \\
& +\left[\begin{array}{llll}
0 & 0 & 0 & 0 \\
0 & \mathbf{K} & 0 & 0 \\
0 & 0 & 0 & 0 \\
0 & 0 & 0 & 0
\end{array}\right]\left\{\begin{array}{c}
\mathbf{d}^{f} \\
\mathbf{d}^{s} \\
0 \\
0
\end{array}\right\}=\left\{\begin{array}{l}
\mathbf{F}_{f} \\
\mathbf{F}_{s} \\
\mathbf{a}_{I} \\
\mathbf{H}
\end{array}\right\},
\end{aligned}
$$

where, $\mathbf{C}_{i j}^{c}$ was the sub-block of fluid-structure coupling matrix. The remaining letter meaning was seen in Ref. [12, 13].

\section{The partitioned algorithm of the coupling of elastic pipe and the cross-flow in cylinder}

The monolithic algorithm is more accurate, the calculation is easier to implement. But the fluid and elastic tube are dispersed by solid element and the model is large which leads the low efficiency in monolithic algorithm. In particular, it is difficult to solve the coupling dynamics problem of tube bundle vibration and collision induced by cross-flow. Based on this, the paper presents a partitioned algorithm for calculating the vibration of elastic tube induced by cross-flow in a cylinder.

In monolithic algorithm, if we do not consider the coupling of cross-flow and elastic tube, the Eq. (7) can be simplified as:

$$
\begin{aligned}
& \left\{\begin{array}{l}
\mathbf{A}_{\alpha \beta} \ddot{\mathbf{d}}^{f}+\left(\mathbf{B}_{\alpha \beta \gamma}^{j} \dot{\mathbf{u}}_{\alpha j}+\mathbf{D}_{\alpha \beta}\right) \cdot \dot{\mathbf{d}}^{f}+\mathbf{C}_{\alpha \beta} \mathbf{p}=\mathbf{F}_{f}, \\
\mathbf{G}_{\alpha \beta} \dot{\mathbf{d}}^{f}=\mathbf{H},
\end{array}\right. \\
& \mathbf{M} \ddot{d}^{s}+\mathbf{C} \dot{d}^{s}+\mathbf{K} d^{s}=\mathbf{F}_{s} .
\end{aligned}
$$

The Eq. (8) is the control equation for fluid domain. The Eq. (9) is the dynamic equation for solid domain of the elastic tube. In partitioned algorithm, it is not necessary to solve the fluid-solid coupling Eq. (7). According to the set order, the Eqs. (8) and (9) are solved respectively. The information which calculated in fluid domain and solid domain are transferred to each other at 
fluid-solid interface (FSI) $[14,15]$. Until the convergence of this time to meet the requirements, then the next moment of the calculation is carried. The final result will be obtained in repeated cycle.

\subsection{The numerical model of the coupling of elastic tube and cross-flow with partitioned algorithm}

The numerical model of the coupling of elastic tube and cross-flow was seen as Fig. 5 with partitioned algorithm. Considering the slender beam characteristics of the elastic tube, the elastic tube was divided into several beam elements to improve the calculation scale and efficiency in partitioned algorithm. The coupling interface between fluid domain and solid domain of the elastic tube was shown in Fig. 6.

It can be seen from Fig. 5 and Fig. 6 that, due to the mismatch between solid element and elastic tube beam element at the coupling interface, the transfer information of displacement, velocity and force was deduced and partitioned algorithm for the coupling of cross-flow and elastic tube in the cylinder is achieved.

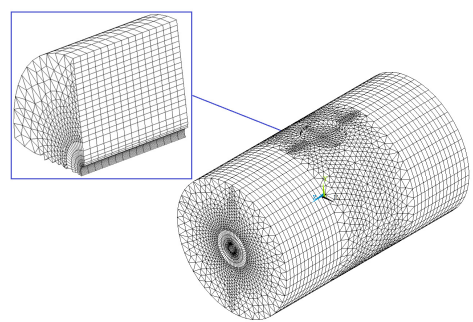

a) The finite model element of fluid domain

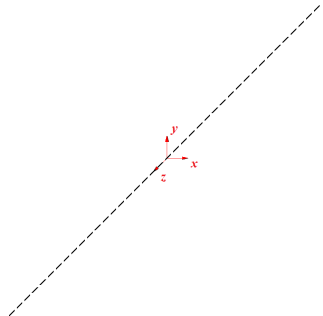

b) The finite element model of elastic tube

Fig. 5. The numerical model of transverse fluid and elastic tube with partitioned method

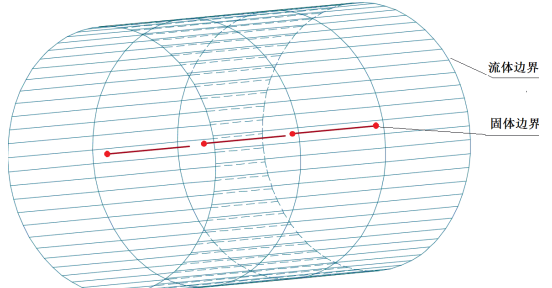

Fig. 6. The coupling interface

\subsection{The information transfer between the elastic pipe and cross-flow in cylinder}

The Fig. 7 showed the transitive relationship between the elastic pipe and cross-flow in cylinder at the coupling interface.

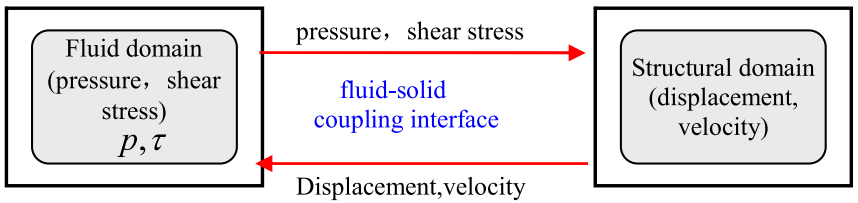

Fig. 7. The transitive relationship between the elastic pipe and cross-flow

The red arrow from the fluid domain to structural domain was represented shear stress and pressure. Namely the fluid affected the elastic pipe by pressure, shear stress. The red arrow from the structural domain to fluid domain was represented displacement and velocity speed. Namely the elastic pipe transmitted displacement and velocity to fluid domain. 


\subsubsection{The information transmission of displacement and velocity at fluid-solid coupling interface}

The information transmission of displacement and velocity was shown in Fig. 8. The beam element named $e$ was taken and its nodes were $i$ and $j$. The vectors of nodal displacement and velocity in local coordinate system were as followed:

$$
\begin{aligned}
& \mathbf{d}_{e}^{s}=\left[\begin{array}{llllllllllll}
u_{i x}^{S} & u_{i y}^{s} & u_{i z}^{s} & \theta_{i x}^{s} & \theta_{i y}^{s} & \theta_{i z}^{s} & u_{j x}^{s} & u_{j y}^{s} & u_{j z}^{s} & \theta_{j x}^{s} & \theta_{j y}^{s} & \theta_{j z}^{s}
\end{array}\right]^{T}, \\
& \dot{\mathbf{d}}_{e}^{s}=\left[\begin{array}{llllllllllll}
\dot{u}_{i x}^{S} & \dot{u}_{i y}^{s} & \dot{u}_{i z}^{s} & \dot{\theta}_{i x}^{s} & \dot{\theta}_{i y}^{s} & \dot{\theta}_{i z}^{s} & \dot{u}_{j x}^{s} & \dot{u}_{j y}^{s} & \dot{u}_{j z}^{s} & \dot{\theta}_{j x}^{s} & \dot{\theta}_{j y}^{s} & \dot{\theta}_{j z}^{s}
\end{array}\right]^{T} \text {. }
\end{aligned}
$$

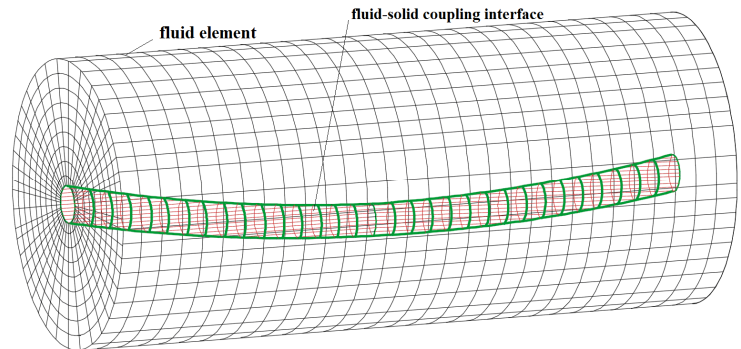

a) The fluid element and fluid-solid coupling interface

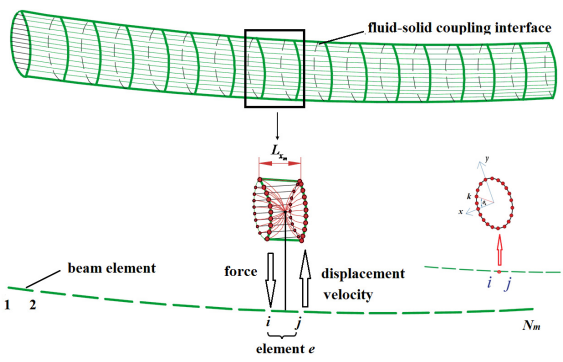

b) The information transmission

Fig. 8. Force, displacement and velocity interpolation at coupling interface

The displacement and velocity of arbitrary axis position $x_{m}$ on the beam element was as followed by shape function $\mathbf{N}$ :

$\mathbf{d}_{e, m}^{s}=\left[\begin{array}{llllll}u_{x, m}^{s} & u_{y, m}^{s} & u_{z, m}^{s} & \theta_{x, m}^{s} & \theta_{y, m}^{s} & \theta_{z, m}^{s}\end{array}\right]^{T}=\left.N\right|_{x=x_{m}} d_{e}^{s}$,

$\dot{\mathbf{d}}_{e, m}^{s}=\left[\begin{array}{llllll}\dot{d}_{x, m}^{s} & \dot{d}_{y, m}^{s} & \dot{d}_{z, m}^{s} & \dot{\theta}_{x, m}^{s} & \dot{\theta}_{y, m}^{s} & \dot{\theta}_{z, m}^{s}\end{array}\right]^{T}=\left.N\right|_{x=x_{m}} \dot{d}_{e}^{s}$.

According to the assumption that the cross section was always circular, the displacement components of the node $k$ at axis position $x_{m}$ was as followed:

$\left\{\begin{array}{l}u_{x, m}^{f}=u_{x, m}^{s}+r \sin \theta_{y, m}^{s} \sin \theta_{k}-r \sin \theta_{z, m}^{s} \cos \theta_{k}, \\ u_{y, m}^{f}=u_{y, m}^{s}+r \cos \left(\theta_{x, m}^{s}+\theta_{k}\right)-r \cos \theta_{k}, \\ u_{z, m}^{f}=u_{z, m}^{s}+r \sin \left(\theta_{x, m}^{s}+\theta_{k}\right)-r \sin \theta_{k},\end{array}\right.$

where, $r$ was outer radius of the elastic pipe. $x_{m}$ was distance from the node $i . \theta_{k}$ was circumferential angle of node $k$, which was shown in Fig. 8.

The displacement vector $\mathbf{d}_{e, m}^{f}$ can be expressed as:

$\mathbf{d}_{e, m}^{f}=\left[\begin{array}{lll}u_{x, m}^{f} & u_{y, m}^{f} & u_{z, m}^{f}\end{array}\right]^{T}=\mathbf{H}_{F S I}^{e} \mathbf{d}_{e, m}^{S}=\left.\mathbf{H}_{F S I}^{e} N\right|_{x=x_{m}} \mathbf{d}_{e}^{S}$,

where, $\mathbf{H}_{F S I}^{e}$ was the displacement transformation matrix from structural domain to fluid domain.

Assigned $\mathbf{H}_{\Gamma_{F S I}}^{e}=\left.\mathbf{H}_{F S I}^{e} N\right|_{x=x_{m}}$, then the displacement, velocity vector of the fluid units on the fluid-solid coupling interface can be expressed as:

$\mathbf{d}_{e, m}^{f}=\mathbf{H}_{\Gamma_{F S I}}^{e} \mathbf{d}_{e}^{S}$,

$\dot{\mathbf{d}}_{e, m}^{f}=\mathbf{H}_{\Gamma_{F S I}}^{e} \dot{\mathbf{d}}_{e}^{s}$.

Thence, the displacement, velocity vector of the fluid at time $t$ can be expressed as: 
$\mathbf{d}_{e, m}^{f}(t)=\mathbf{H}_{\Gamma_{F S I}}^{e}(t) \mathbf{d}_{e}^{s}(t)$,

$\dot{\mathbf{d}}_{e, m}^{f}(t)=\mathbf{H}_{\Gamma_{F S I}}^{e}(t) \dot{\mathbf{d}}_{e}^{s}(t)$.

When the elastic pipe was deformed, according to the Eq. 15(a) and 15(b), the displacement and velocity would be re-applied on the boundary wall in fluid domain. Therefore, the coordination of displacement and velocity was achieved at the fluid-solid coupling interface.

\subsubsection{The information transmission of load at the fluid-solid coupling interface}

As shown in Fig. 9, the interface loads were obtained at the outer surface of the elastic pipe in fluid domain. And the loads were decomposed into normal pressure $p$, tangential shear stress $\tau_{r \phi}$ and axial shear stress $\tau_{r x}$. First, loads were integrated along the surface and simplified at the corresponding axis. Then according to the principle of virtual work, loads were equivalent to the values of nodes $i, j$ of beam element.

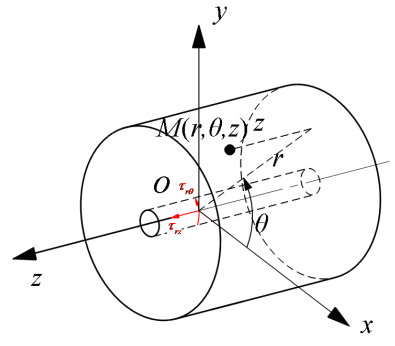

a) Space

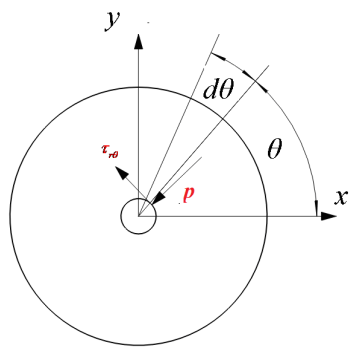

b) Plane

Fig. 9. The interface loads at the outer surface of the elastic pipe in fluid domain

In the beam element at $x=x_{m}$, the length of element was $L_{x_{m}}$. The forces and torques was simplified was as followed:

$$
\left\{\begin{array}{l}
q_{F_{x}}^{m}=\int_{0}^{L_{x_{m}}} \int_{0}^{2 \pi}\left(\tau_{r \theta} \sin \theta+P \cos \theta\right) d \theta d z, \\
q_{F_{y}}^{m}=\int_{0}^{L_{x_{m}}} \int_{0}^{2 \pi}\left(\tau_{r \theta} \cos \theta+P \sin \theta\right) d \theta d z, \\
q_{F_{z}}^{m}=\int_{0}^{L_{x_{m}}} \int_{0}^{2 \pi} \tau_{r z} d \theta d z \\
q_{m_{x}}^{m}=r \int_{0}^{L_{x_{m}}} \int_{0}^{2 \pi} \tau_{r \theta} \sin \theta d \theta d z, \\
q_{m_{y}}^{m}=r \int_{0}^{L_{x_{m}}} \int_{0}^{2 \pi} \tau_{r \theta} \cos \theta d \theta d z, \\
q_{m_{z}}^{m}=r \int_{0}^{L_{x_{m}}} \int_{0}^{2 \pi} \tau_{r z} d \theta d z .
\end{array}\right.
$$

The Eq. (16) was represented by a vector:

$\mathbf{q}_{m}^{f}=\left[\begin{array}{llllll}q_{F_{x}}^{m} & q_{F_{y}}^{m} & q_{F_{z}}^{m} & q_{m_{x}}^{m} & q_{m_{y}}^{m} & q_{m_{z}}^{m}\end{array}\right]^{T}$.

According to the principle of virtual work, the above-mentioned axial loads were further equivalent to values of the node on beam elements: 
$\mathbf{F}_{e}^{f}=\sum_{m=1}^{n_{f o}^{x}} \mathbf{F}_{f}^{i}=\sum_{m=1}^{n_{f o}^{x}} \mathbf{T}^{T} \int_{0}^{L_{x_{m}}} \mathbf{N}^{T} \mathbf{q}_{m}^{f} d x$,

where, $\mathbf{T}$ was the converted matrix of coordinates.

All nodal loads which were equivalent to the beam element were assembled. Then, the equivalent nodal loads $\mathbf{F}_{f}(t)$ were obtained from the fluid domain to the elastic pipe. Thus, it met the conditions of load balancing at the coupling interface.

Substituted $\mathbf{F}_{f}(t)$ into the dynamic Eq. (9) of the elastic pipe, the dynamic equations of the elastic pipe was obtained as followed when considering the effect of fluid:

$\mathbf{M} \ddot{\mathbf{d}}^{s}+\mathbf{C} \dot{\mathbf{d}}^{s}+\mathbf{K} \mathbf{d}^{s}=\mathbf{F}_{s}+\mathbf{F}_{f}$,

where, the $\mathbf{F}_{f}$ was the vector of fluid force at the coupling interface.

\subsection{The convergence criterion of coupling of elastic pipe and cross-flow in the cylinder}

In the coupling, iterative step of fluid domain $\Omega_{f}(t)$ and solid domain $\Omega_{s}(t)$, the normalized convergence criterion at coupling interface at any one time step was as followed:

$\varepsilon_{\Gamma i}^{j_{k}}(t)=\frac{\left\|\xi_{\Gamma i}^{\text {new }}(t)-\xi_{\Gamma i}^{j_{k}}(t)\right\|_{2}}{\left\|\xi_{\Gamma i}^{\text {new }}(t)\right\|_{2}}<\varepsilon_{\Gamma i}^{0}, \quad i=f, s$,

where, $t$ was the current time step. $j k$ was the iteration number at the current time step. $i=f, s$ were represented the fluid-solid coupling interface of fluid domain and structural domain respectively.

The physical quantity of fluid-structure coupling interface was $\boldsymbol{\xi}_{\Gamma i}(t)=\left\{\begin{array}{llll}\mathbf{d}_{\Gamma i}(t) & \mathbf{u}_{\Gamma i}(t) & \mathbf{F}_{\Gamma i}(t)\end{array}\right\}^{T}$. Where, $\mathbf{d}_{\Gamma i}(t), \quad \mathbf{u}_{\Gamma i}(t)$ and $\mathbf{F}_{\Gamma i}(t)$ were vectors of displacement, velocity and force at the coupling interface in fluid domain or structural domain. $\xi_{\Gamma i}^{j_{k}}(t)$ was the physical quantity which was applied at current iteration step. $\xi_{\Gamma i}^{n e w}(t)$ was the new physical quantity. $\varepsilon_{\Gamma i}^{j_{k}}(t)$ was the normalized convergence value. $\varepsilon_{\Gamma i}^{j_{k}}(t)=\left\{\begin{array}{lll}\varepsilon_{d \Gamma i}^{j_{k}} & \varepsilon_{u \Gamma i}^{j_{k}} & \varepsilon_{F \Gamma i}^{j_{k}}\end{array}\right\}^{T}$. Where, $\varepsilon_{\Gamma i}^{0}=\left\{\begin{array}{ccc}\varepsilon_{d \Gamma i}^{0} & \varepsilon_{u \Gamma i}^{0} & \varepsilon_{F \Gamma i}^{0}\end{array}\right\}^{T}$, which was the convergence tolerance.

\subsection{The frame diagram of partitioned algorithm for elastic pipe and cross-flow in cylinder}

The Fig. 10 was the total frame diagram of partitioned algorithm. $t_{\text {end }}$ was the total computational time. The iterative process was as followed.

(1) The models of fluid domain and structural domain were established. Give inlet velocity $u$ and outlet pressure $P$. Give the initial displacement and initial velocity at the coupling interface.

(2) Solve the fluid domain $\Omega_{f}$ and get the coupling interface load $F_{\Gamma f}^{j k}(t)$;

(3) According to the Eq. (18), get the coupling interface loads $\mathbf{F}(t)$ of structural domain;

(4) Solve structural domain $\Omega_{s}$, get displacement and velocity at coupled interface in structural domain.

(5) According to the Eq. (15), get the displacement $\mathbf{d}_{\Gamma s}^{j k}(t)$ and velocity $\mathbf{u}_{\Gamma s}^{j k}(t)$ at coupled interface in fluid domain.

(6) According to the convergence Eq. (20), if the values of displacement, velocity and load do not meet the convergence criteria, return the coupled iteration from the Eq. (3) to Eq. (6).

(7) If the values of displacement, velocity and load meet the convergence Eq. (20) at the same time, Order $t=t+\Delta t$, follow the previous coupling step Eq. (2) to (6). Otherwise, end of the 
iteration.

The coupling iteration loop at the current time step was seen as the Fig. 11.

In partitioned algorithm of the elastic pipe and the cross-flow in cylinder, the data of displacement, velocity and load was transferred with the time. The process of time-step cycle was seen in Fig. 12.

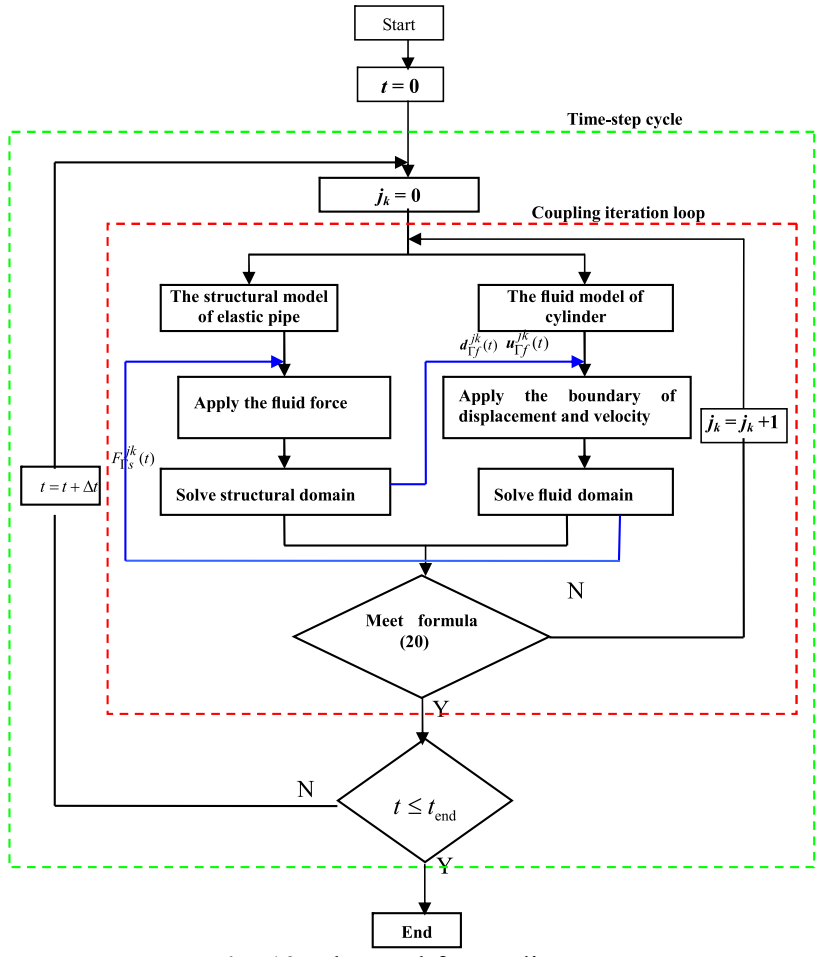

Fig. 10. The total frame diagram of partitioned algorithm

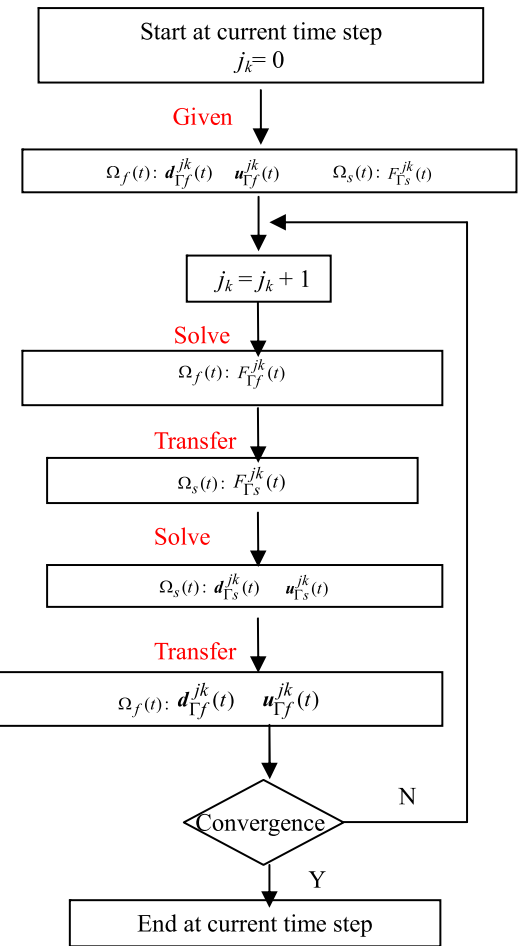

Fig. 11. The calculation process at the current time step

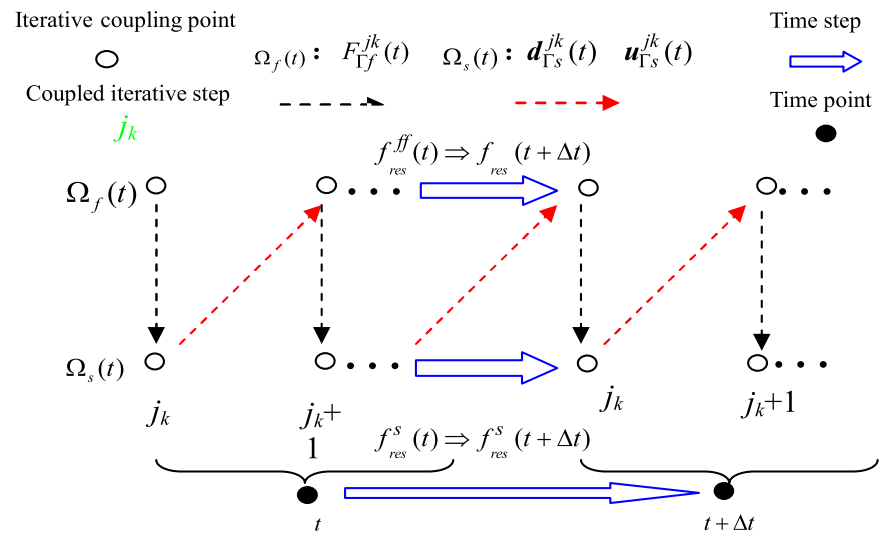

Fig. 12. The process of time-step cycle

Seen from the Fig. 12, the results files $f_{\text {res }}^{f}(t), f_{\text {res }}^{s}(t)$ of fluid domain and structural domain were used as initial values at time $t$. Then solving the fluid domain and structural domain at time $t+\Delta t$, the results files $f_{\text {res }}^{f}(t+\Delta t), f_{\text {res }}^{s}(t+\Delta t)$ were obtained. And the $F_{\Gamma f}(t+\Delta t)$ in fluid 
domain, the $\mathbf{d}_{\Gamma f}(t+\Delta t)$ and $\mathbf{u}_{\Gamma f}(t+\Delta t)$ in structural domain were taken at the same time. Thus, these physical quantities would be initial values at time $t+\Delta t$.

\section{Examples and verification}

\subsection{Cases and calculation parameters}

The calculation parameters of the elastic pipe and fluid were listed in Table.

Table 1. The calculation parameters of the elastic pipe and fluid

\begin{tabular}{|l|c|}
\hline Length $(\mathrm{m})$ & 0.5 \\
\hline Outer diameter $(\mathrm{m})$ & 0.02 \\
\hline Inner diameter $(\mathrm{m})$ & 0.016 \\
\hline Elastic modulus $(\mathrm{GPa})$ & 3.5 \\
\hline Poison's ratio & 0.3 \\
\hline The density of elastic pipe $\left(\mathrm{kg} / \mathrm{m}^{3}\right)$ & 2250 \\
\hline The density of fluid $\left(\mathrm{kg} / \mathrm{m}^{3}\right)$ & 1000 \\
\hline Dynamic viscosity $(\mathrm{mPa} \cdot \mathrm{s})$ & 1 \\
\hline Inlet velocity $(\mathrm{m} / \mathrm{s})$ & 2.236 \\
\hline outlet pressure $(\mathrm{MPa})$ & 0 \\
\hline Natural frequency of elastic pipe $(\mathrm{Hz})$ & 23.91 \\
\hline Calculation time step $(\mathrm{s})$ & 0.001 \\
\hline
\end{tabular}

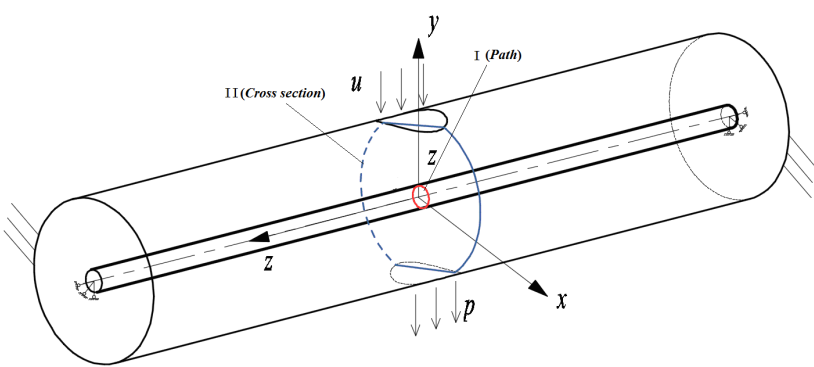

Fig. 13. The path I and cross section II in the model

As shown in Fig. 1, the elastic was placed at the center in cylinder. The elastic pipe in the cylinder fluid was calculated by the monolithic and partitioned algorithm respectively. Then the vortex-induced vibration of the elastic was obtained in solid domain. And the distribution of the pressure and velocity were also obtained in fluid domain. The corresponding results were compared by the two calculation methods. The path I and cross section II of the elastic pipe was shown in Fig. 13. The contrast results of the elastic pipe in solid domain were shown in Fig. 14 and Fig. 15 and the values were listed in Table 2 along the path I. The contrast results in fluid domain were shown in Fig. 16 and Fig. 17 and the values were listed in Table 3 at cross Section 2.

\subsubsection{The contrast results of elastic pipe in solid domain.}

Seen from Fig. 14(a), (b) and (c), the curves of the acceleration, velocity, displacement were identical by the methods of monolithic and partitioned for the elastic pipe. From Table 2, the maximum error of the acceleration amplitude was $5.33 \%$ at time $0.0 \mathrm{~s}-0.3 \mathrm{~s}$. And the maximum error of the velocity amplitude was $4.90 \%$ for the elastic pipe. And the maximum error of the displacement amplitude was $4.20 \%$. All error is less than $5 \%$. Hence, it presented the correctness of the partitioned algorithm for the coupling of the elastic pipe and the cross-flow in cylinder. 


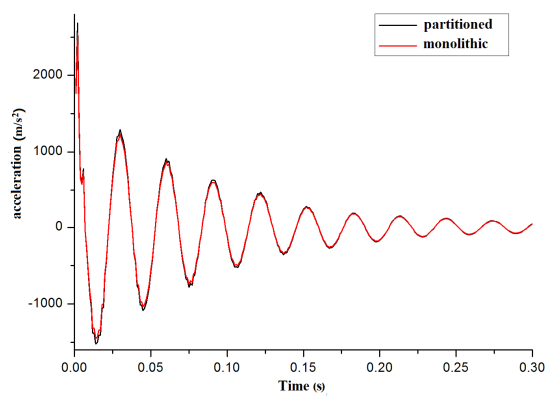

a) Acceleration

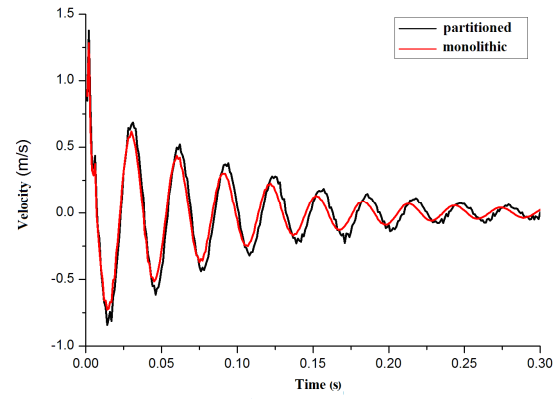

b) Velocity

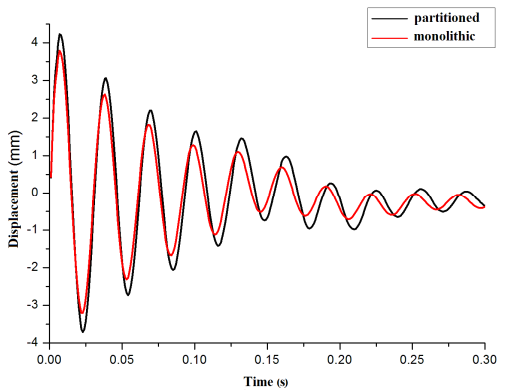

c) Displacement

Fig. 14. The contrast curves of acceleration, velocity, displacement for the elastic pipe

Table 2. The amplitude list of the acceleration, velocity, displacement

\begin{tabular}{|c|c|c|c|c|c|c|c|}
\hline \multicolumn{3}{|c|}{ Time period (s) } & $0-0.03$ & $0.03-0.06$ & $0.06-0.09$ & $0.09-0.12$ & $0.12-0.15$ \\
\hline \multirow{3}{*}{ Acceleration } & \multirow{2}{*}{$\begin{array}{c}\text { Amplitude } \\
\left(\mathrm{m} / \mathrm{s}^{2}\right)\end{array}$} & Monolithic & 4006 & 2267 & 1615 & 1101 & 771 \\
\hline & & Partitioned & 4015 & 2272 & 1632 & 1118 & 786 \\
\hline & \multicolumn{2}{|c|}{ Error $(\%)$} & 0.22 & 0.22 & 1.05 & 1.54 & 1.94 \\
\hline \multirow{3}{*}{ Velocity } & \multirow{2}{*}{$\begin{array}{c}\text { Amplitude } \\
(\mathrm{mm} / \mathrm{s})\end{array}$} & Monolithic & 2011 & 1130 & 790 & 555 & 395 \\
\hline & & Partitioned & 2079 & 1152 & 798 & 561 & 401 \\
\hline & \multicolumn{2}{|c|}{ Error $(\%)$} & 3.38 & 1.95 & 1.01 & 1.08 & 1.52 \\
\hline \multirow{3}{*}{ Displacement } & \multirow{2}{*}{$\begin{array}{c}\text { Amplitude } \\
\text { (mm) }\end{array}$} & Monolithic & 7.32 & 5.59 & 4.03 & 2.99 & 2.09 \\
\hline & & Partitioned & 7.90 & 5.79 & 4.23 & 3.05 & 2.19 \\
\hline & \multicolumn{2}{|c|}{ Error $(\%)$} & 7.92 & 3.58 & 4.96 & 2.01 & 4.78 \\
\hline \multicolumn{3}{|c|}{ Time period (s) } & $0.15-0.18$ & $0.18-0.21$ & $0.21-0.24$ & $0.24-0.27$ & $0.27-0.30$ \\
\hline \multirow{3}{*}{ Acceleration } & \multirow{2}{*}{$\begin{array}{c}\text { Amplitude } \\
\left(\mathrm{m} / \mathrm{s}^{2}\right)\end{array}$} & Monolithic & 514 & 348 & 251 & 197 & 150 \\
\hline & & Partitioned & 521 & 352 & 258 & 203 & 158 \\
\hline & \multicolumn{2}{|c|}{ Error $(\%)$} & 1.36 & 1.15 & 2.79 & 3.05 & 5.33 \\
\hline \multirow{3}{*}{ Velocity } & \multirow{2}{*}{$\begin{array}{l}\text { Amplitude } \\
(\mathrm{mm})\end{array}$} & Monolithic & 235 & 186 & 132 & 102 & 82 \\
\hline & & Partitioned & 239 & 189 & 136 & 107 & 85 \\
\hline & \multicolumn{2}{|c|}{ Error $(\%)$} & 1.70 & 1.61 & 3.03 & 4.90 & 3.66 \\
\hline \multirow{3}{*}{ Displacement } & \multirow{2}{*}{$\begin{array}{c}\text { Amplitude } \\
\left(\mathrm{m} / \mathrm{s}^{2}\right)\end{array}$} & Monolithic & 1.86 & 1.19 & 0.70 & 0.60 & 0.39 \\
\hline & & Partitioned & 1.92 & 1.24 & 0.72 & 0.62 & 0.42 \\
\hline & \multicolumn{2}{|c|}{ Error $(\%)$} & 3.23 & 4.20 & 2.86 & 3.33 & 7.69 \\
\hline
\end{tabular}

\subsubsection{The contrast results of pressure and velocity distribution in fluid domain}

The distribution of pressure and velocity was shown as Fig. 15 along path I in fluid domain at $t=0.281 \mathrm{~s}$.

Seen from Fig. 15, the distribution of pressure and velocity was identity by the algorithm of monolithic and partitioned coupling along path I. Of note, in $48^{\circ}-132^{\circ}$, namely in the position of upstream face, the fluid pressure was positive, greater than atmospheric pressure and the maximum value was at the position of $90^{\circ}$. In $132^{\circ}-210^{\circ}, 330^{\circ}-360^{\circ}, 0^{\circ}-48^{\circ}$, namely in the 
position of side face, the fluid pressures were relatively negative, less than atmospheric pressure and the maximum negative value was occurred in $174^{\circ}$ and $6^{\circ}$ position. In $220^{\circ}-320^{\circ}$, namely in the position of lee face, the fluid pressures were relatively positive, greater than atmospheric pressure. But the values were smaller than values of upstream face.

Seen from the Table 3, the errors of pressure calculated by the two methods were $-0.17 \%$, $0.04 \%-0.87 \%, 0.15 \%$ respectively, in the location of $6^{\circ}, 90^{\circ}, 174^{\circ}, 27^{\circ}$ along path $\mathrm{I}$. And the errors of velocity were $-0.27 \%, 1.71 \%,-0.27 \%,-1.35 \%$ respectively. Therefore, the values of pressure and velocity obtained by the two methods were equivalent in the fluid domain.

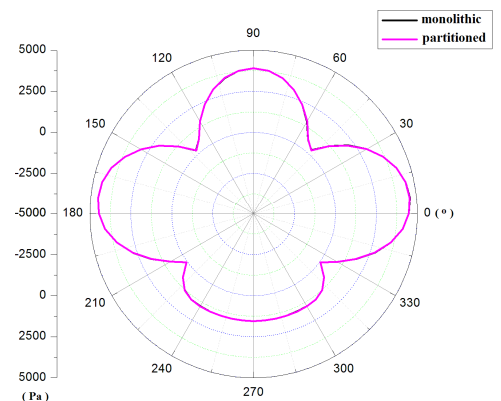

a) Pressure

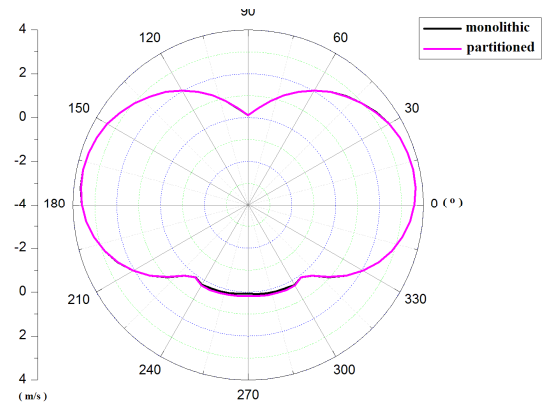

b) Velocity

Fig. 15. The pressure and velocity distribution along path I

Table 3. The list of the pressure and velocity along path I

\begin{tabular}{|c|c|c|c|c|c|c|}
\hline \multirow{2}{*}{ Positions $\left({ }^{\circ}\right)$} & \multicolumn{3}{|c|}{ Pressure } & \multicolumn{3}{c|}{ Velocity } \\
\cline { 2 - 7 } & Monolithic (Pa) & Partitioned (Pa) & Error (\%) & Monolithic (m/s) & Partitioned (m/s) & Error (\%) \\
\hline 6 & -4589.70 & -4581.91 & -0.17 & -3.73 & -3.72 & -0.27 \\
\hline 90 & 3896.77 & 3898.17 & 0.04 & -0.115 & -0.117 & 1.71 \\
\hline 174 & -4590.43 & -4550.63 & -0.87 & -3.68 & -3.67 & -0.27 \\
\hline 270 & 1552.97 & 1555.29 & 0.15 & 0.075 & 0.074 & -1.35 \\
\hline
\end{tabular}

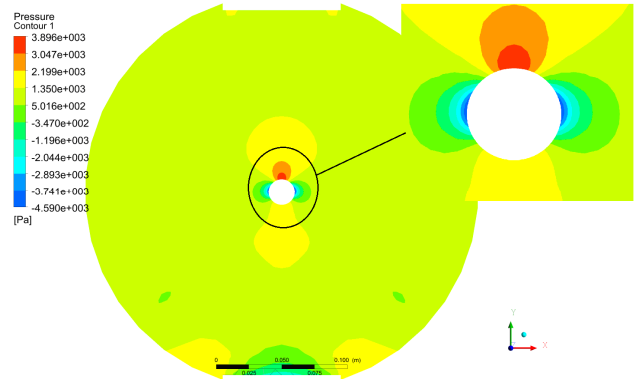

a) Pressure

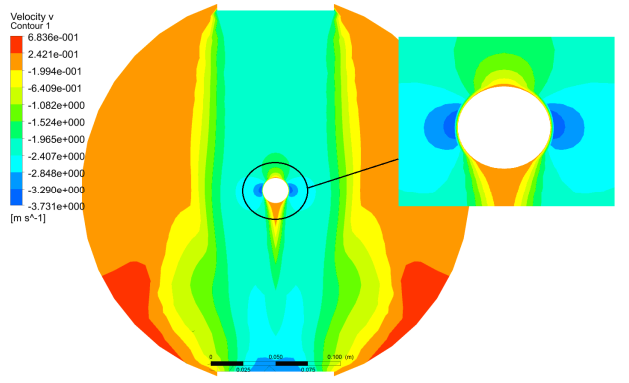

b) Velocity

Fig. 16. The pressure and velocity contours at cross section II by monolithic algorithm

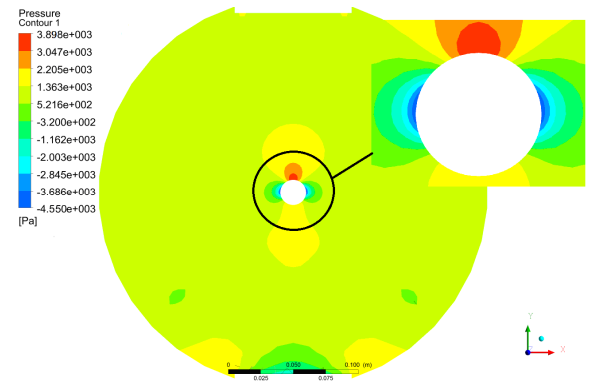

a) Pressure

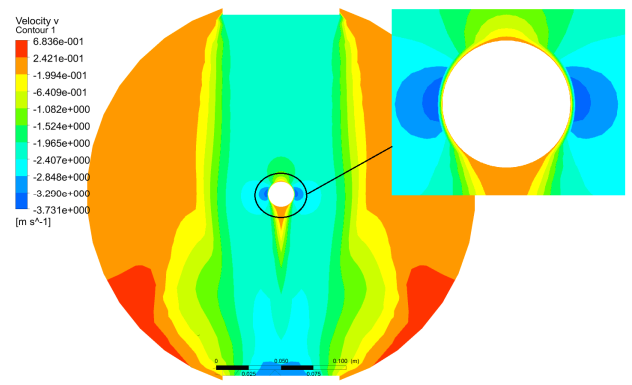

b) Velocity

Fig. 17. The pressure and velocity contours at cross Section 2 by partitioned algorithm 
The Fig. 16 and Fig. 17 were pressure and velocity contours at cross Section 2 by monolithic and partitioned algorithm. From the contours, the distribution of pressure and velocity at cross Section 2 were basically the same.

\section{Conclusions}

1) The coupling mechanics model was established for the elastic pipe and the cross-flow in cylinder. The boundary conditions of the inlet flow rate, outlet pressure and wall were considered in the model. And it also met the conditions of displacement, velocity and load balancing at fluid-structure coupling interface.

2) In monolithic algorithm, the model of fluid and elastic tube is both dispersed by solid elements. The dynamic coupling finite element equation was applied to solve the dynamic characteristics of elastic pipe in solid domain and the velocity and pressure in fluid domain. Although the monolithic algorithm is easy and accurate, the model is very lager in general and it may lead a low efficiency. In particular, it is difficult to solve the coupling dynamics problem of multiple bundles vibration and collision induced by cross-flow in a more complex fluid domain. Based on this, the partitioned algorithm was established for elastic pipe and cross-flow in cylinder. In partitioned algorithm, the elastic pipe was separated into several beam elements. The cross-flow was still separated into solid elements. At the fluid-structure coupling interface, the formulas of displacement, velocity and load was derived and the corresponding convergence criterion was also derived. The iterative block diagram of partitioned coupling algorithm was designed.

3) According the above two methods, the example was calculated by the algorithm of monolithic and partitioned. The results proved that the curves of acceleration, velocity, displacement for elastic pipe were consistent by two algorithms in solid domain. And pressure and velocity distribution were also consistent by two algorithms at cross section II in fluid domain. It proved the correctness of the partitioned coupling algorithm which was established in this article.

4) The method will provide an effective computational method for vibrations and collision of multiple bundles in a more complex fluid domain. And it will have important theoretical significance in engineering.

\section{Acknowledgements}

This work was supported by the Project of National Natural Science Foundation of China (11272085).

\section{References}

[1] Tang You Gang, Fan Juan Juan, Zhang Jie In line and transverse vortex-induced vibration analysis for a circular cylinder under high Reynolds number. Journal of Vibration and Shock, Vol. 36, Issue 13, 2013, p. 88-92.

[2] Ji Jia Dong, Ge Pei Qi, Bi Wen Bo Numerical analysis on the combined flow induced vibration response of elastic to tube bundle in heat exchange. Journal of Xi'an Jiao Tong University, Vol. 45, Issue 4, 2015, p. 24-29.

[3] Ragulskis M., Fedaravicius A., Ragulskis K. Harmonic balance method for FEM analysis of fluid flow in a vibrating pipe. Communications in Numerical Methods in Engineering, Vol. 22, Issue 4, 2006, p. 347-356.

[4] Heil M., Hazel Boyle A. L. J. Solvers for large-displacement fluid - structure interaction problems: segregated versus monolithic approaches. Computational Mechanics, Vol. 43, Issue 1, 2008, p. 91-101.

[5] Yan Ke, Ge Peiqi, Bi Wenbo, et al. Vibration characteristics of fluid-structure interaction of conical spiral tube bundle. Journal of Hydrodynamics, Vol. 22, Issue 1, 2010, p. 121-128.

[6] Antoci Carla, Gallati Mario, Sibilla Stefano Numerical simulation of fluid-structure interaction by SPH. Computers and Structures, Vol. 85, Issues 11-14, 2007, p. 879-890.

[7] Zhang A., Suzuki K. A comparative study of numerical simulmions for fluid-structure interaction of liquid-filled tank during ship collision. Ocean Engineering, Vol. 34, Issue 5, 2007, p. 645-652. 
[8] Ali Eken, Mehmet Sahin A parallel monolithic algorithm for the numerical simulation of large-scale fluid structure interaction problems. International Journal for Numerical Methods in Fluids, Vol. 80, Issue 12, 2016, p. 687-714.

[9] Bathe K. J., Zhang H. Finite element developments for general fluid flows with structural interactions. International Journal for Numerical Methods in Engineering, Vol. 60, Issue 1, 2004, p. 213-232.

[10] Longatte E., Verreman V., Souli M. Time marching for simulation of fluid-structure interaction problems. Journal of Fluids and Structures, Vol. 25, Issue 1, 2009, p. 95-111.

[11] Hu Shiliang, Lu Chuanjing, He Yousheng Fluid-structure interaction simulation of threedimensional elastic hydrofoil in water tunnel. Applied Mathematics and Mechanics, Vol. 37, Issue 1, 2016, p. 15-26.

[12] Fabian D., Raul G., Srinivasan N. Arbitrary Lagrangian-Eulerian method for Navier-Stokes equations with moving boundaries. Computer Methods in Applied Mechanics and Engineering, Vol. 193, Issues 45-47, 2004, p. 4819-4836.

[13] Liu Yun He Transient Dynamic Analysis of Fluid-Structure Coupling on the Application on Theory and Hydraulic Engineering. Xi' an Jiao Tong University, 2001, p. 76-78.

[14] Yue Qian Bei, Dong Ri Zhi, Liu Ju Bao The research of vortex-induced vibration for the elastic pipe at different locations in a limited fluid domain. Applied Mathematics and Mechanics, Vol. 37, Issue 3, 2016, p. 277-289.

[15] Liu Ju Bao, Luo Min, Wang Lin Methodology of fluid-structure interaction of rotary slender beam pipe. Engineering Mechanics, Vol. 28, Issue 4, 2011, p. 251-256.

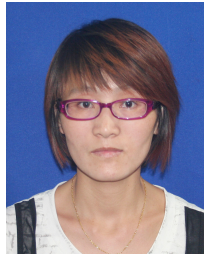

Qian Bei Yue received Ph.D. in mechanical engineering from Northeast Petroleum University, DaQing City, China, in 2014. Now she works at Northeast Petroleum University. Her current research interests include columns' mechanics and fluid-structure.

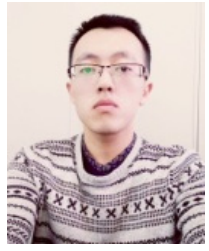

Ri Zhi Dong received Bachelor's degree in engineering mechanics from Northeast Petroleum University, Daqin, China, in 2015. Now he is working for Master's degree.

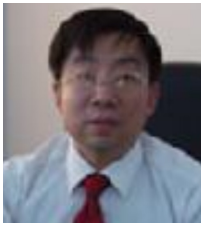

Ju Bao Liu received Ph.D. in Mechanical Engineering from ZheJiang University, Hangzhou city, China, in 1999. Now he works at Northeast Petroleum University. His current research interests include columns's mechanics, fluid-structure and vibration control. 